\title{
Quantitative Evaluation of DNA-Binding Data In Vivo for Low-Dose Extrapolations
}

\author{
W. K. Lutz \\ Institute of Toxicology, ETH and University of Zurich, CH-8603 Schwerzenbach, Switzerland
}

\section{Introduction}

The risk of tumor formation from exposure to a chemical carcinogen is dependent on the exposure, the potency of the carcinogen, and the irfividual host reaction. Humans are exposed to chemical carcinogens at dose levels which are orders of magnitude below the levels used in"animal studies on carcinogenicity. The latter experiments provide significant data only at high-dose levels which lead to tumor incidences on the order of percent (Fig. 1). For humans, tolerable exposures producing not more than one additional tumor in one million lives should be defined. The extrapolation range therefore covers four to five orders of magnitude. Instead of using purely mathematical models for the extrapolation, it would be desirable to have a biologically relevant indicator which could be investigated in the dose range to be bridged.

A large group of chemical carcinogens is known to bind covalently to DNA in the target cell. Under appropriate conditions, this primary DNA lesion can be expressed as a mutation finally leading to cancer. The primary interaction of the carcinogen with DNA can be investigated with appropriate techniques (radiolabeled test compound, phosphorylation with ${ }^{32} \mathrm{P}$, or immunological methods) at low dose levels which would not give rise to a detectable increase in tumor yield with a limited number of animals treated. It is therefore possible to investigate the shape of the dose-response curve in the region of interest.

\section{Methods}

With radiolabeled test compound, the limit of detection is dependent on the specific activity, on the binding potency (covalent binding index, CBI; Lutz 1979), on the amount of DNA analyzed, and on the radioactivity in a vial considered significant. Under optimal conditions met, for instance, with tritiated aflatoxin $\mathrm{B}_{1}$ of $8 \mathrm{Ci} / \mathrm{mmol}$ and a $\mathrm{CBI}$ of 10000 , a single dose of $1 \mathrm{ng} / \mathrm{kg}$ rat was the observed limit of detection for liver DNA binding (10 dpm net radioactivity in $6 \mathrm{mg}$ DNA). 


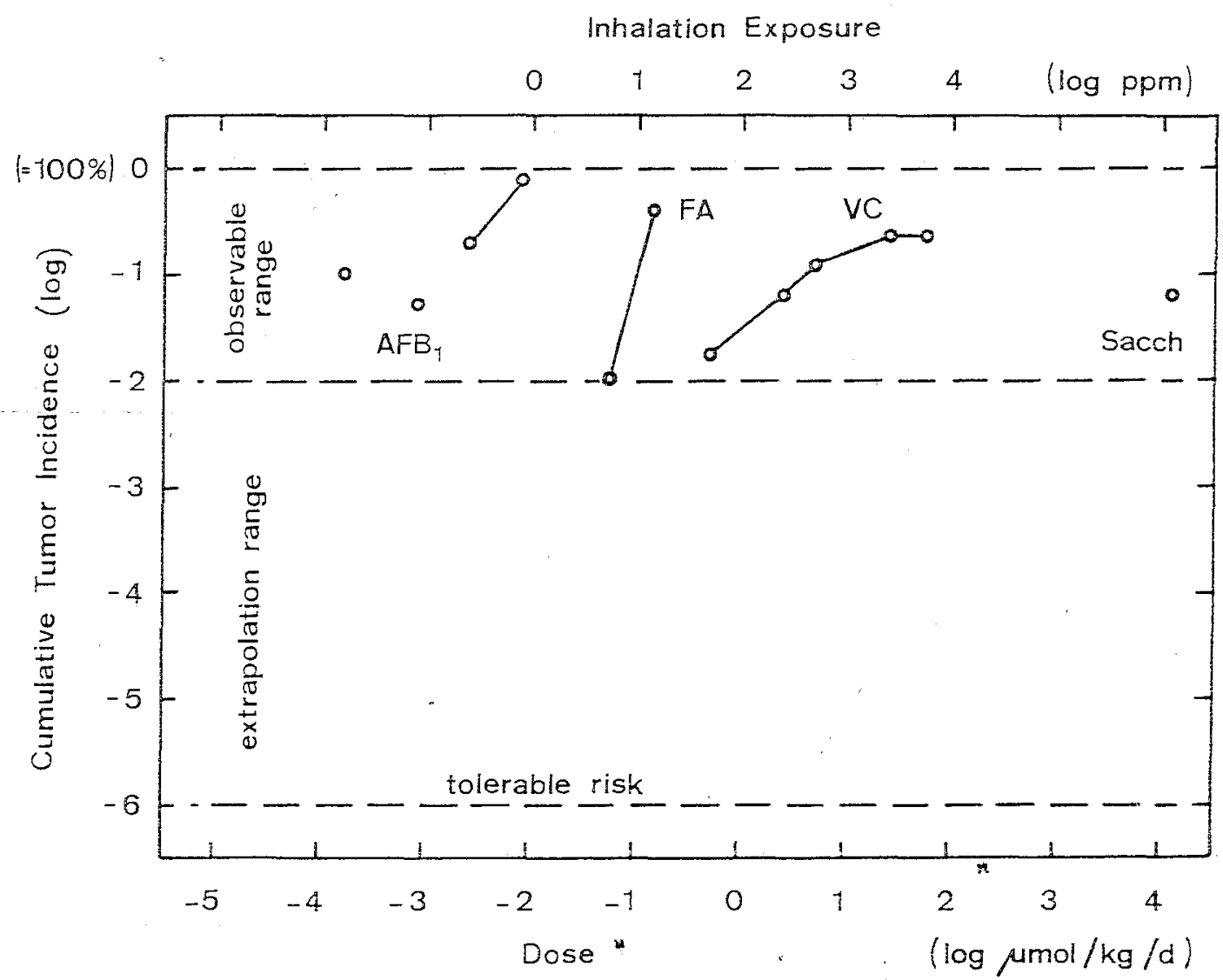

Fig. 1. Cumulative tumor incidence in rats as a function of the level of exposure. For aflatoxin $B_{1}$ $\left(A F B_{1}\right)$ and saccharin (Sacch) the compound was admixed to the diet; the bottom dose scale applies. For formaldehyde $(F A)$ and vinyl chloride $(V C)$ exposure was by inhalation, $6 \mathrm{~h} /$ day, 5 days/week, for 2 years; the top dose scale applies. The two scales were combined on the assumption of a respiratory minute volume of $750 \mathrm{ml}$ air $/ \mathrm{kg}$ and an absorption of $20 \%$

This corresponded to 3 adducts per $10^{11}$ nucleotides, i.e., less than 1 adduct per liver cell genome. With ${ }^{14} \mathrm{C}$-labeled compounds of $10 \mathrm{mCi} / \mathrm{mmol}$, the limit of detection would have been about 500 times higher, i.e., 1 adduct in $10^{8}$ nucleotides.

A model calculation for the postlabeling technique using HPLC methods gives the following results: With $\left[\gamma^{32} \mathrm{P}\right] \mathrm{ATP}$ of a specific activity of $1000 \mathrm{Ci} / \mathrm{mmol}$, an assumed limit of detection of $20 \mathrm{dpm}$ would correspond to $0.01 \mathrm{fmol}$ adduct, i.e., a level of 3 in $10^{9}$ nucleotides in a $1-\mu \mathrm{g}$ DNA sample.

With antibodies and the slot-blot technique (Rajewsky, personal communication), $0.1 \mathrm{fmol}$ adduct can be detected in $3 \mu \mathrm{g}$ DNA. This corresponds to an adduct level of 1 in $10^{8}$ nucleotides.

A comparison of the three methods therefore shows that for the testing of new compounds, and where only small amounts of DNA are available from a specific tissue, the postlabeling technique seems most versatile. 


\section{Theoretical Considerations of the Shape of the Dose-Response Curve for DNA Binding}

Many of the DNA-binding carcinogens require metabolic activation to form electrophilic, reactive, so-called ultimate carcinogens. DNA binding therefore involves enzymatic processes, diffusion processes, and electrophilic substitution reactions. In principle, the rate of all these steps is proportional to the concentration in the low range, so that a linear dose-DNA binding relationship would be postulated. The rate of DNA repair has been found to be proportional to the level of the DNA adducts in the case of $0^{6}$-methylguanine (Lutz 1982). If this first order kinetics for DNA repair holds also for other adducts, a linear dose-DNA binding relationship would hold not only for the time of maximum binding, but also at later times of DNA analysis.

\section{Experimental Findings on the Dose-DNA Binding Relationship}

A number of compounds have been studied in this respect. The dose range investigated is shown in Fig. 2. A small arrow indicates for each compound the $T_{50}$

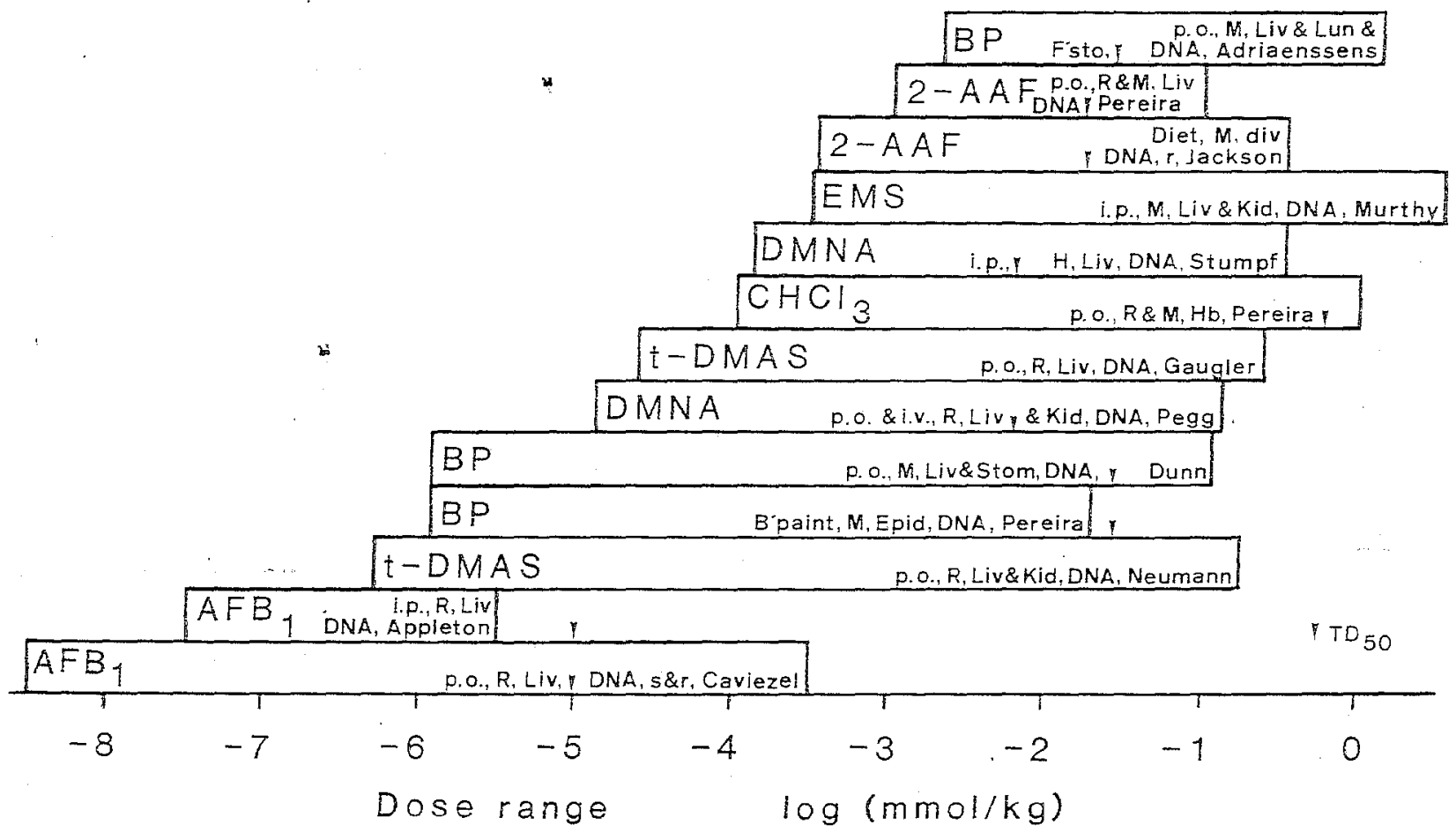

Fig. 2. Range of doses investigated with various carcinogens for macromolecular binding in vivo. The small arrows indicate the $\mathrm{TD}_{50}$ value (Gold et al. 1984), i.e., a measure for carcinogenic potency. Abbreviations of substances from left to right: $A F B_{1}$, aflatoxin $\mathrm{B}_{1} ; t-D M A S$, trans-4-dimethylaminostilbene; $B P$, benzo[a]pyrene; $D M N A$, dimethylnitrosamine; $E M S$, ethyl methanesulfonate; 2- $A A F$, 2-acetylaminofluorene. Other abbreviations indicate the route of exposure, the animal species, the organ of interest, the type of macromolecule, and a specification of single $(s)$ or repeated $(r)$ administration: $R$, rat; $M$, mouse; $H$, hamster; Liv, liver; kid, kidney; Epid, epidermis; Stom, stomach; Lun, lung; F'sto, forestomach; div, diverse organs; $H b$, hemoglobin. The respective reference is listed under the first a.uthor's name. (From P. Buss, Ph.D. thesis, ETH Zurich, in preparation) 


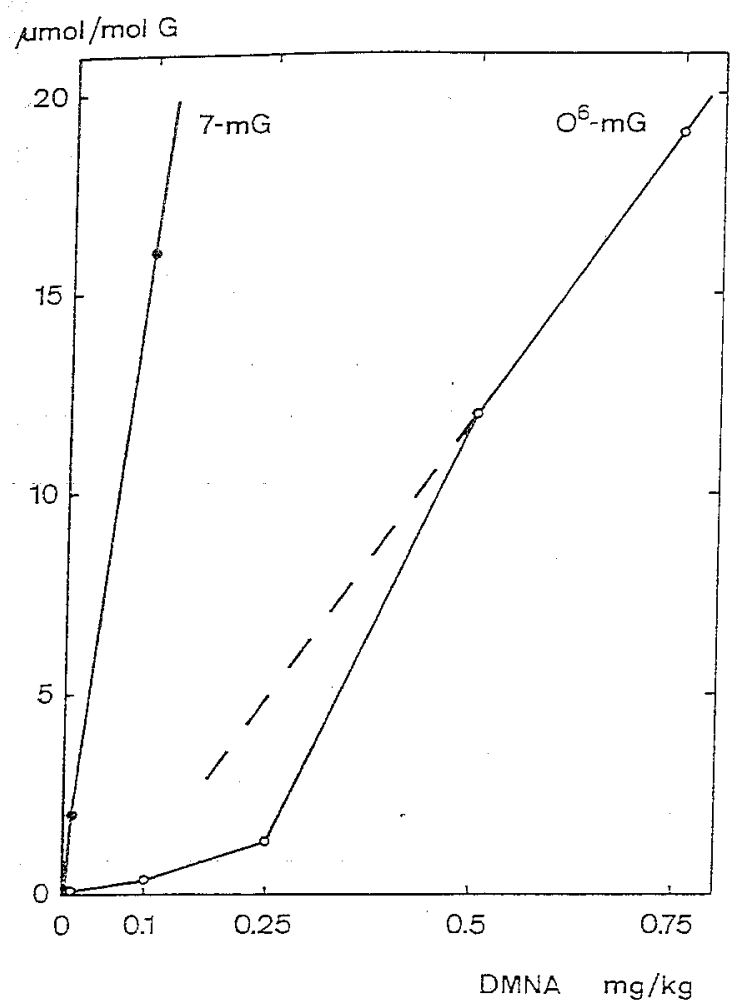

Fig. 3. Dose-response curve for the determination of 7-methylguanine $(7-m G)$ and $O^{6}$-methylguanine $\left(O^{6}-m G\right)$ in liver DNA isolated from male Syrian golden hamsters, $5 \mathrm{~h}$ after i.p. injection of radiolabeled dimethylnitrosamine (DMNA). (Original data from Stumpf et al. 1979)

value (Gold et al. 1984), i.e., the approximate daily dose required to induce a tumor in $50 \%$ of the animals treated. With respect to the problem of extrapolation of tumor-inducing doses to lower exposures, those studies are the most relevant which cover three or more orders of magnitude below the $\mathrm{TD}_{50}$. Studies which did not extend beyond one order of magnitude below the $\mathrm{TD}_{50}$ were not included in the list. All studies except those investigating specific methylated DNA bases showed a linear dose-response curve. The lowest dose used was $1 \mathrm{ng} / \mathrm{kg}$ with aflatoxin $B_{1}$ (Caviezel 1984). The theoretical prediction of proportionality could therefore be verified experimentally.

The only situation showing a nonlinearity is exemplified in Fig. 3. The formation of 7-methylguanine increased linearly with the DNNA dose, whereas the level of $O^{6}$-methylguanine was proportional to the dose only above $0.5 \mathrm{mg} / \mathrm{kg}$. Below that dose, the level of $O^{6}$-methylguanine was lower than extrapolated from the high dose levels. This phenomenon was indicative of a fast-acting repair process which was exhausted at higher levels of $O^{6}$-methylguanine (Lutz 1982). For this adduct it could also mean that a linear extrapolation of the tumor incidence data to lower doses might be too conservative because a small DNA damage might be more quickly repaired than a large one. A nonlinear doseresponse relationship results. Since DNA methylation so far is the only DNA damage leading to this type of dose response it is possible that this constitutive, fast repair of erroneous DNA methylations has evolved in nature in order to control errors in the formation of 5-methylcytidine by the endogenous methyl donor $S$-adenosylmethionine. 
It is becoming evident that DNA binding is nonrandom with respect both to sequence and arrangement of the DNA. This does interfere with the quantitative relationship of total DNA binding to the number of critical mutations, but it should not affect the analysis of the dose-response relationship because it can be assumed that the fraction of critical adducts will be proportional to the total number of adducts.

\section{Singie Vs Repetitive Exposure}

In a standard carcinogenicity bioassay, the test compound is given continuously. The level of DNA adducts therefore is a result of a constant daily increase from new exposure and the removal by repair, cell death, or cell division. What is decisive for the tumorigenicity of DNA adducts in the steady-state level. Only two studies have been dealing with the low dose-response relationship for DNA binding after repetitive exposure.

1. Various concentrations of 2-acetylaminofluorene (2-AAF) were admixed to the diet fed for 2 weeks to mice of different strains. DNA was isolated from different organs. The level of DNA adducts was strictly proportional to the concentration of 2-AAF in the diet (Jackson et al. 1980).

2. In this laboratory, tritiated aflatoxin $B_{1}$ was administered p.o. to F344 rats on 10 consecutive days (Caviezel 1984). Liver DNA was isolated $24 \mathrm{~h}$ after the last dose and the level of DNA tamage was determined. Again, a linear dose-response relationship was found over 4 orders of magnitude (Fig. 4). The damage set by the highest dose was no longer proportional to the dose, probably because of a saturation of the activating enzyme systems. This phenomenon was also observed with DNA adducts induced by trans-4-dimethylaminostilbene (t-DMAS; Neumann 1980) and vinyl chloride (Watanabe et al. 1978) and is reflected in earcinogenicity studies by a flattening out of the dose-response curve at the highest exposure levels (see Fig. 1 vinyl chloride, VC).

\section{Conciusions on DNA-Binding in Exposed Individuals}

The data summarized above indicate that the level of genotoxicity is proportional to the dose in the low dose range after both single and repetitive administration of a DNA-binding compound. In the high dose range, a flattening out of the curve can be seen if the metabolic activation is becoming saturated.

The lowest dose used so far for a DNA-binding study was $1 \mathrm{ng}$ aflatoxin $\mathrm{B}_{1}$ administered per $\mathrm{kg}$ rat. This corresponds to about $60 \mathrm{ng}$ per man, i.e., a dose which is taken up daily in certain areas of tropical Africa and Asia. The lowest point shown in Fig. 4 therefore represents a dose to which humans can be exposed.

For risk extrapolation of DNA-binding carcinogens it therefore seems most appropriate to underline the idea that the primary lesion is strictly proportional to the dose. The nonlinearity seen with $0^{6}$-methylguanine is one exception to the rule and probably cannot be used for other types of adducts. 


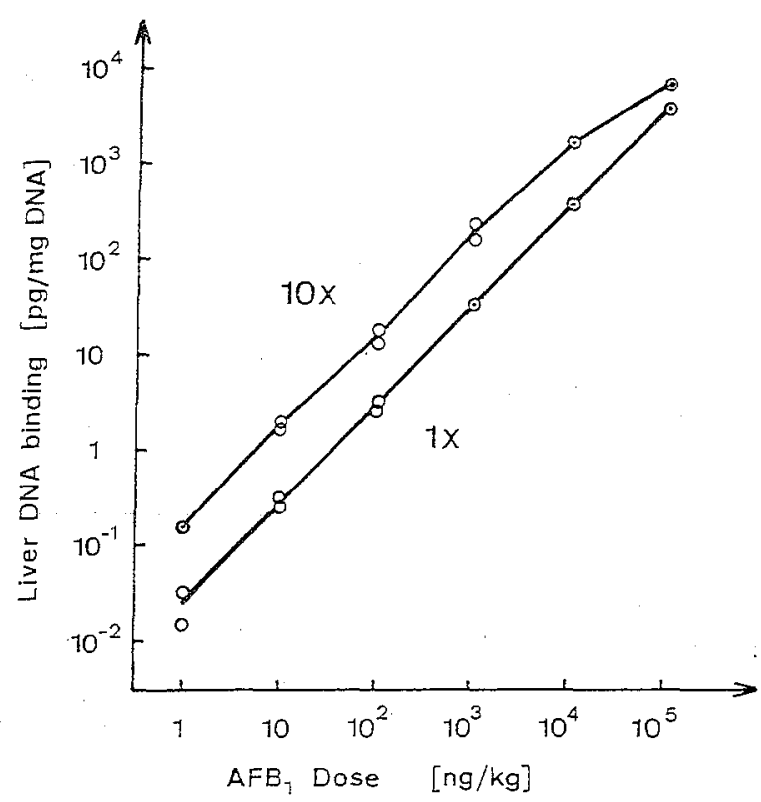

Fig. 4. Binding of tritiated aflatoxin $\mathrm{B}_{1}\left(A F B_{1}\right)$ to liver DNA of male F344 rats, $24 \mathrm{~h}$ after one single $(1 x)$ or the last of ten daily oral doses $(10 x)$, as a function of the dose

\section{Low-Dose Extrapolations in Populations}

Both theoretical analysis and experimental data indicate that, for the primary lesion in chemical carcinogenesis by DNA-binding compounds, a linear extrapolation is appropriate. This proportionality cannot, however, be extended to the question of the dose-response relationship for the appearance of tumors in a population without additional considerations. In general toxicology it is very often seen that the distribution of individuals giving a predetermined response (yes or no criterion) to an agent is gaussian (normal) if the doses are scaled logarithmically. This log-normal distribution can be derived exactly from the reasonable pharmacological assumption that the effect will become observable if the concentration of the toxic principle is above a certain limit during a critical period of time. If it is further accepted that the individuals in the population differ according to a normal distribution with respect to either the critical time period necessary or the rate of detoxication, the values of a minimal effective concentration will be log-normally distributed (Koch 1966).

An element of time is therefore required for the logarithm to enter into the dose-response relationship. Time is also of predominant importance in the process of chemical carcinogenesis, but here it is not a matter of an all or none response, but rather of a stochastic succession of stages where the probability is dependent on the period of time available. For instance, a heritable mutation can only be derived from a DNA adduct if the DNA is replicating to produce a genetic alteration in a daughter cell. The fixation of the primary lesion is therefore dependent on the relative rates of DNA repair and DNA replication. A reduction of the time available for the mutational expression of the DNA adduct will therefore reduce the probability of a mutation.

Many mathematical risk models take into account that carcinogenesis is a multistage process with several hits being required with or without specific sequence 
assumptions. The observed tumor incidences are used to determine best-fit values of the parameters of the underlying function. If such functions are used for lowdose extrapolations, an unresolvable problem arises from the assumption that the host reaction to the high dose levels also determines the shape of the dose-response curve at lower dose levels (FDA 1971).

\section{Dose-Response Relationship for Combination Mechanisms of Carcinogenic Action}

DNA binding is only one out of a number of activities resulting in increased tumor formation (Lutz 1986). Cell division seems to be an absolute requirement in carcinogenesis, and agents or processes which stimulate the rate of DNA synthesis are often found to increase the tumor incidence. Some compounds most probably produce DNA adducts and stimulate cell division: hexachlorocyclohexane isomers could be named (Sagelsdorff et al.1983) and formaldehyde might also be an example (Swenberg et al. 1983). In both cases it is considered that the DNAbinding activity is low so that it would not alone be sufficient to lead to a significant increase in tumor formation in a standard bioassay (Fig. 5, dashed line). Only together with sufficient promotion would an effect become observable. With formaldehyde, this promoting activity - in a mechanistic sense - could be due to cytotoxicity and tissue regeneration. Since cell death is an "all or nothing" phenomenon, discussed above," as the basis of a log-normal dose-response relationship, a nonlinear curve could result. A combination of DNA binding with stimulation of cell division would still be nonlinear (Fig. 5). The steep slope observed for the tumor incidence from formaldehyde inhalation exposure to 5.6 and $14.3 \mathrm{ppm}$ (Fig.1) could therefore be explained by a combination of direct genotoxicity and tissue irritation.

The slope shown for aflatoxin $B_{1}$ and vinyl chloride (low dose part only) is approximately one in the double-log plot. The proportionality of dose and DNA

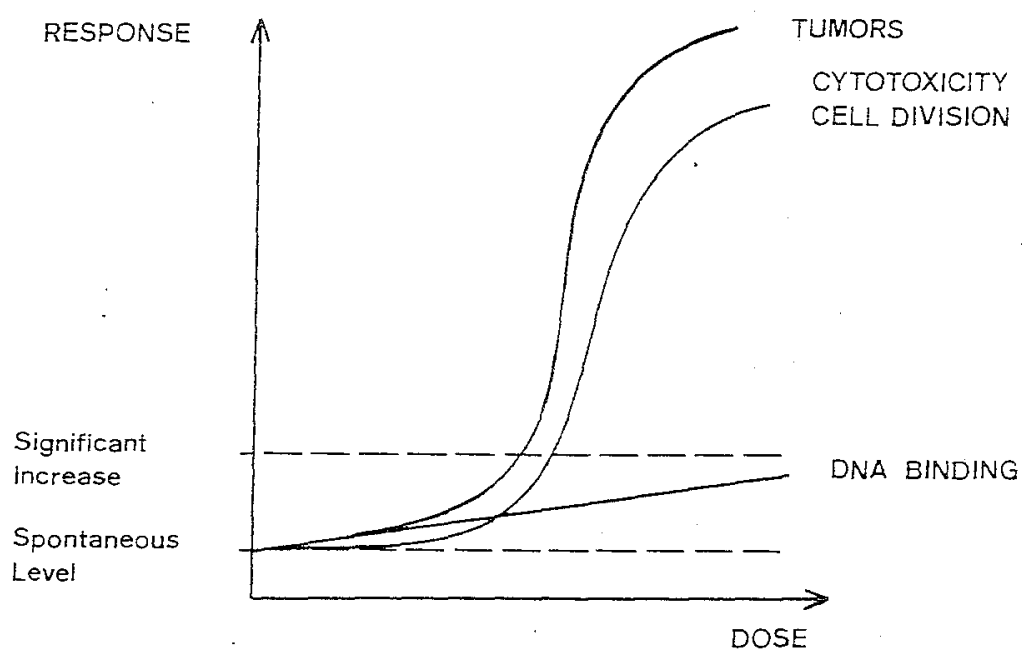

Fig. 5. Potential dose-response relationship for a chemical carcinogen exhibiting more than one biological activity related to tumor induction. Cytotoxicity with regenerative cell division is given as one possible example for a promoting type activity 
binding as discussed above therefore seems to determine the dose-response relationship for the tumor incidence. This could mean that DNA binding is the predominant factor in chemical carcinogenesis by $\mathrm{AFB}_{1}$ and VC. DNA binding is only one mechanism of genotoxicity. Stimulation of cell division is only one aspect in tumor promotion. The present analysis of the available data shows that these two aspects might explain tumor formation by a large number of chemicals. Nevertheless, other types of activities might be involved in the process of carcinogenesis. Such critical biochemical processes should be searched for.

The present discussion has shown that the assessment of the risk of exposure to a tumor-inducing agent at low dose levels could be improved if mechanisms of action were taken into account. Low-dose extrapolations should therefore be based more often on biological models. Neither standard carcinogenicity bioassays nor mathematical models provide such information.

Acknowledgement. We thank the Swiss League Against Cancer for financial support (FOR 267 AK 84/5).

\section{References}

Adriaenssens PI, White CM, Anderson MW (1983) Dose-response relationships for the binding of benzo[a]pyrene metabolites to DNA and protein in lung, liver, and forestomach of control and butylated hydroxyanisole-treated mice. Cancer Res 43:3712-3719

Appleton BS, Goetchius MP, Campbell TC (1982) Linear dose-response curve for the hepatic macromolecular binding of aflatoxin $B_{1}$ in ratsuat very low exposure. Cancer Res 42:3659-3662

Caviezel M (1984) Untersuchungen zur kovalenten Bindung an DNS und Protein in vivo durch die Steroide Cholesterin-5,6-epoxid, Oestron, Oestradiol and Trenbolon, sowie durch Aflatoxin $B_{1}$. Ph.D. Thesis, ADAG Zürich, Diss. ETH Nr. 7564

Dunn BP (1983) Wide-range linear dose-response curve for DNA binding of orally administered benzo[a]pyrene in mice. Cancer Res 43:2654-2658

FDA (1971) Food and drug administration advisory committee on protocols for safety evaluation: panel on carcinogenesis report on cancer testing in the safety evaluation of food additives and pesticides. Toxicol Appl Pharmacol 20:419-438

Gaugler BJM, Neumann HG (1979) The binding of metabolites formed from aminostilbene derivatives to nucleic acids in the liver of rats. Chem-Biol Interact 24:355-372

Gold LS, Sawyer CB, Magaw R, Backman GM, de Veciana M, Levinson R, Hooper NK, Havender WR, Bernstein L, Peto R, Pike MC, Ames BN (1984) A carcinogenic potency database of the standardized results of animal bioassays. Environ Health Perspect 58:9-319

Jackson CD, Weis C, Shellenberger TE (1980) Tissue binding of 2-acetylaminofluorene in BALB/c and C57Bl/6 mice during chronic oral administration. Chem-Biol Interact 32:63-81

Koch AI (1966) The logarithm in biology: 1. Mechanisms generating the log-normal distribution exactly. J Theor Biol 12:276-290

Lutz WK (1979) In vivo covalent binding of organic chemicals to DNA as a quantitative indicator in the process of chemical carcinogenesis. Mutat Res 65:289-356

Lutz WK (1982) Inducible repair of DNA methylated by carcinogens. Trends Pharmacol Sci 3:398399

Lutz WK (1986) Quantitative evaluation of DNA binding data for risk estimation and for classification of direct and indirect carcinogens. J Cancer Res Clin Oncol 112:85-91

Murthy MSS, Calleman CJ, Osterman-Golkar S, Segerbäck D, Svensson K (1984) Relationship between ethylation of hemoglobin, ethylation of DNA and administered amount of ethyl methanesulfonate in the mouse. Mutat Res 127:1-8

Neumann HG (1980) Dose-response relationship in the primary lesion of strong electrophilic carcinogens. Arch Toxicol [Suppl] 3:69-77

Pegg AE, Perry W (1981) Alkylation of nucleic acids and metabolism of small doses of dimethylnitrosamine in the rat. Cancer Res 41:3128-3132 
Pereira MA, Burns FJ, Albert RE (1979) Dose response for benzo[a]pyrene adducts in mouse epidermal DNA. Cancer Res 39:2556-2559

Pereira MA, Lin LHC, Chang LW (1981) Dose-dependency of 2-acetylaminofluorene binding to liver DNA and hemoglobin in mice and rats. Toxicol Appl Pharmacol 60:472-478

Pereira MA, Chang LW (1982) Binding of chloroform to mouse and rat hemoglobin. Chem-Biol Interact 39:89-99

Sagelsdorff P, Lutz WK, Schlatter C (1983) The relevance of covalent binding to mouse liver DNA for the carcinogenic action of hexachlorocyclohexane isomers. Carcinogenesis 4:1267-1273

Stumpf R, Margison GP, Montesano R, Pegg AE (1979) Formation and loss of alkylated purines from DNA of hamster liver after administration of dimethylnitrosamine. Cancer Res 39:50-54

Swenberg JA, Barrow CS, Boreiko CJ, Heck HA, Levine RJ, Morgan KT, Starr TB (1983) Non-linear biological responses to formaldehyde and their implications for carcinogenic risk assessment. Carcinogenesis 4:945-952

Watanabe PG, Zempel JA, Pegg DG, Gehring PJ (1978) Hepatic macromolecular bincing following exposure to vinyl chloride. Toxicol Appl Pharmacol 44:571-579 